\title{
Computer-aided detection of breast lesions in DCE-MRI using region growing based on fuzzy C-means clustering and vesselness filter
}

\author{
Shahriar B. Shokouhi ${ }^{1 *}$, Aida Fooladivanda ${ }^{1}$ and Nasrin Ahmadinejad ${ }^{2}$
}

\begin{abstract}
A computer-aided detection (CAD) system is introduced in this paper for detection of breast lesions in dynamic contrast-enhanced magnetic resonance imaging (DCE-MRI). The proposed CAD system firstly compensates motion artifacts and segments the breast region. Then, the potential lesion voxels are detected and used as the initial seed points for the seeded region-growing algorithm. A new and robust region-growing algorithm incorporating with Fuzzy C-means (FCM) clustering and vesselness filter is proposed to segment any potential lesion regions. Subsequently, the false positive detections are reduced by applying a discrimination step. This is based on 3D morphological characteristics of the potential lesion regions and kinetic features which are fed to the support vector machine (SVM) classifier. The performance of the proposed CAD system is evaluated using the free-response operating characteristic (FROC) curve. We introduce our collected dataset that includes 76 DCE-MRI studies, 63 malignant and 107 benign lesions. The prepared dataset has been used to verify the accuracy of the proposed CAD system. At 5.29 false positives per case, the CAD system accurately detects 94\% of the breast lesions.
\end{abstract}

Keywords: Breast DCE-MRI, Computer-aided detection, Lesion detection, FCM, Vesselness filter

\section{Introduction}

Breast cancer is the most frequent type of cancer and the second leading cause of cancer deaths among women worldwide [1]. Dynamic contrast-enhanced magnetic resonance imaging (DCE-MRI) is an established medical imaging technique in screening, diagnosis, and staging of breast cancer. MRI produces cross-sectional images of a breast with higher sensitivity compared to mammography and ultrasound. Additionally, this imaging modality does not use ionizing radiation as opposed to X-ray mammography $[2,3]$. However, interpretation of 4D DCE data is needed to analyze breast MRI. Evaluation of the enormous amount of images for each patient is a time-consuming process, and it depends on a radiologist's expertise and experience [4]. Moreover, some important details which could affect the

\footnotetext{
* Correspondence: bshokouhi@iust.ac.ir

${ }^{1}$ School of Electrical Engineering, Iran University of Science and Technology (IUST), Tehran, Iran

Full list of author information is available at the end of the article
}

final diagnosis may be missed. Computer-aided detection (CAD) systems are introduced to help the radiologist for analyzing biomedical data [5-7]. A breast MRI CAD system that marks suspicious locations of a breast can avoid overlooked or misinterpreted lesions and reduce the analysis time.

A limited number of studies deal with the automatic detection of lesions in breast DCE-MRI. The method developed by Ertas et al. [8] segments breast regions using a cellular neural network and detects lesions by performing $3 \mathrm{D}$ template matching on the normalized maximum intensity-time ratio maps. Renz et al. [9] suggest applying a hierarchical 3D Gaussian pyramid method to segment breast lesions. Vignati et al. [10] discover breast lesions using a normalization technique based on the contrast-uptake of mammary vessels. Chang et al. [11] utilize kinetic and 3D morphological features to spot focal tumor breast lesions.

All of the mentioned studies report the detection rate only for mass-like lesions without considering non- 
mass-like enhancing lesions. According to the BI-RADS breast MRI lexicon [12], a mass is a 3D space-occupying lesion. Comparatively, the enhancement of an area that is not a mass refers to a non-mass-like enhancing lesion. The detection of non-mass-like enhancing lesions is a vital issue, because a large number of breast lesions have non-mass-like enhancement. It is shown that $20-30 \%$ of all invasive lesions and majority of the DCIS lesions are non-mass-like enhancement [13]. To date, only GubernMérida et al. [14] offer an automated approach for localizing both mass and non-mass-like enhancing lesions in breast DCE-MRI. In their method, lesion candidates are located based on the blob and relative enhancement voxel features. Then, the region-based morphological and kinetic features are utilized to identify the malignancy score of lesion candidates. They only report malignant lesions as a result of their CAD system.

The target of this study is to implement an automated CAD system for detecting both benign and malignant breast lesions with mass-like or non-mass-like enhancement in DCE-MRI. A new and robust seeded regiongrowing algorithm based on the vesselness filter and Fuzzy C-means (FCM) clustering method is proposed for the segmentation of any potential lesion regions. This is the first time that FCM clustering and vesselness filter are incorporated in the seeded region-growing algorithm. The proposed method begins by correcting motion artifact and segmenting breast region. Subsequently, the voxels that might belong to breast lesions are segmented out and taken as initial seed points of the region-growing algorithm. Finally, the spurious candidate regions are removed by $3 \mathrm{D}$ morphological characteristics and kinetic features. Free-response operating characteristic (FROC) curve is utilized to evaluate the performance of the presented CAD system with the prepared database of manually annotated benign and malignant lesions which include both mass-like and non-mass-like enhancement. This paper is arranged into the following sections. In Section 2, the clinical breast MRI scans used in our research are introduced. After explaining the proposed CAD system in Section 3, the experimental results are discussed in Section 4. Finally, the conclusions of the research outcomes are presented in Section 5.

\section{Breast MRI database}

The breast MRI scans from 76 women, with ages between 25 and 69 years and average of 48 , are used in this study to verify the accuracy of the proposed CAD system. The cases are collected from Noor medical imaging center in Tehran between January 2012 and January 2016. Because of questionable abnormalities in previous mammogram and/or ultrasound, these women undergo breast MRI. Ethics approval is obtained from the
Institutional Review Board, and informed consent is waived. Breast MRI scanning process is performed in the prone position with a dedicated four-channel breast coil (CP breast Array, Siemens) on a 1.5 Tesla Siemens scanner (Magnetom, Simphony). The axial T1-weighted breast volumes are acquired by a 3D fast low angle shot (FLASH) pulse sequence. The clinical imaging parameters are as follows: matrix size $=320 \times 320$ or $448 \times 448$ or $512 \times 512$, field of view $=370-430 \mathrm{~mm}$, flip angle $=$ $14^{\circ}$, repetition time $(\mathrm{TR})=4.7 \mathrm{~ms}$, echo time $(\mathrm{TE})=$ $1.5 \mathrm{~ms}$, and slice thickness $=2-2.8 \mathrm{~mm}$. Fifty-six axial slices are utilized to cover the entire breast. The DCEMRI data sets are acquired before and after a bolus injection of $0.1 \mathrm{mmol} / \mathrm{kg}$ of contrast agent (Gd-DTPA) with an interval of $75 \mathrm{~s}$. The first sequence is taken before an intravenous agent injection followed by five post-contrast series.

The prepared dataset includes 107 benign and $63 \mathrm{ma}-$ lignant lesions which are confirmed by histopathological examinations or supported by clinical follow-up. From a total number of lesions included in this study, 125 lesions have mass-like enhancement and 45 lesions have non-mass-like enhancement. For our dataset, the lesion radius ranges from 2.50 to $37.18 \mathrm{~mm}$ with an average of $12.25 \mathrm{~mm}$ and standard deviation of $9.75 \mathrm{~mm}$. The benign lesions are 51 fibrocystic changes, 35 papillomas, and 21 fibroadenomas. The malignant lesions contain 35 invasive ductal carcinomas (IDC), 19 ductal carcinoma in situ (DCIS), and 9 invasive lobular carcinomas (ILC). An expert radiologist, with more than 5 years of experience in breast DCE-MRI examination and diagnosis, retrospectively annotates the lesions in a dedicated breast DCE-MRI annotation environment [15]. The reports are the basis for identifying size, location, and mass-like or non-mass-like enhancement of the lesions. The lesion segmentation process is manually performed on the subtraction volume which is generated by subtracting the image intensities of the first post-contrast sequence from the pre-contrast sequence. The prepared dataset (IUST-BREASTMRI-DATASET1) is available in our website: http://een.iust.ac.ir/profs/Shokouhi/IUSTBREASTMRI-DATABASE.

\section{Methodology}

The block diagram of the proposed CAD system for the automatic detection of breast lesions in DCE-MRI is presented in Fig. 1. As the first processing steps, motion artifacts are corrected and the breast region is segmented. Then, the potential lesion voxels are detected and utilized as the initial seed points for region-growing algorithm. Subsequently, the region-growing method based on FCM clustering algorithm and vesselness filter segments the potential lesion regions. Eventually, a discrimination step is used by relying on the morphological 


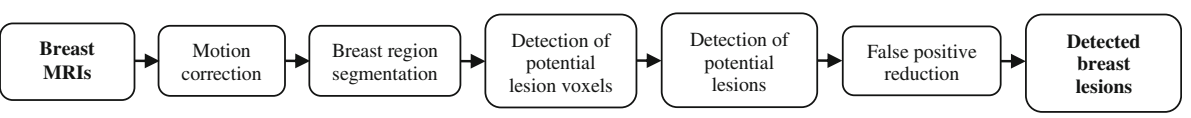

Fig. 1 Block diagram of the proposed CAD system for detection of breast lesions in DCE-MRI

and kinetic features as inputs to the support vector machine (SVM) classifier to reduce false positives detections. The processing steps are sequentially explained in the following subsections.

\subsection{Motion correction}

Respiration, cardiac motion, muscle relaxation, and involuntary movements of a patient cause motion artifacts which are inevitable due to relatively long acquisition period of breast DCE-MRI. Motion artifacts can influence the lesion kinetic characteristics and also increase false positive findings by generating spurious enhancing voxels. In this research, motion correction is performed by registering all five post-contrast sequences to the pre-contrast sequence. The registration step contains a rigid transformation followed by a non-rigid B-Spline transformation [16] based on mutual information similarity measure [17]. The rigid transformation which contains translation and rotation provides global alignment of two images. Likewise, the local differences between the images are minimized by non-rigid transformation. For rigid registration, 1000 iterations of the stochastic gradient descent optimizer have been performed. For non-rigid B-spline registration, three resolutions and 64 histogram bins have been utilized and the gradient descent optimization algorithm is applied in each resolution performing 200 iterations. The registration process has been implemented using medical image registration toolbox (MIRT) [18]. The influence of motion correction for a sample subtracted image at the fifth postcontrast time point is presented in Fig. 2.

\subsection{Breast region segmentation}

Breast region segmentation is automatically performed to decrease the computational burden and avoid false positive findings due to enhancing tissues of the heart and vessels outside of the breast. The segmentation procedure in [19] is applied to extract the breast region. The breast segmentation pipeline consists of four consecutive stages: local adaptive thresholding, connected component labeling operation to exclude the extra regions in the binary image, horizontal projection to delineate the breast region, and both hole-filling and morphological closing operators to eliminate the discontinuities in the breast region. The breast segmentation approach has been applied on nonfat-suppressed images due to high signal intensity of fat tissue that makes a high contrast between adjacent regions. Hence, the breast boundary is detected by applying a prior registration and then translating the obtained breast masks in nonfat-suppressed images to the subtracted images. Figure 3 shows breast region segmentation method using sample images. The breast region is firstly detected on the nonfat-suppressed image, and then, the obtained breast mask is translated to the subtracted image by applying the registration method.

\subsection{Detection of potential lesion voxels}

The next step is to segment out the voxels that might belong to the breast lesions. Following injection of contrast agent, enhancement of signal intensity occurs in all breast lesions except in cysts. Therefore, the enhancing voxels in post-contrast sequences are dubious to be a part of breast lesions. For detecting potential lesion voxels, the maximum enhancement ratio is utilized using the following equation [8]:

$$
M E(x, y, z)=\max \left(\frac{I_{t}(x, y, z)-I_{0}(x, y, z)}{I_{0}(x, y, z)}, t=1,2, \cdots, 5\right),
$$

where $I_{0}$ and $I_{\mathrm{t}}$ are the intensity values on pre-contrast and $t^{t h}$ post-contrast sequences, respectively, and $(x, y, z)$
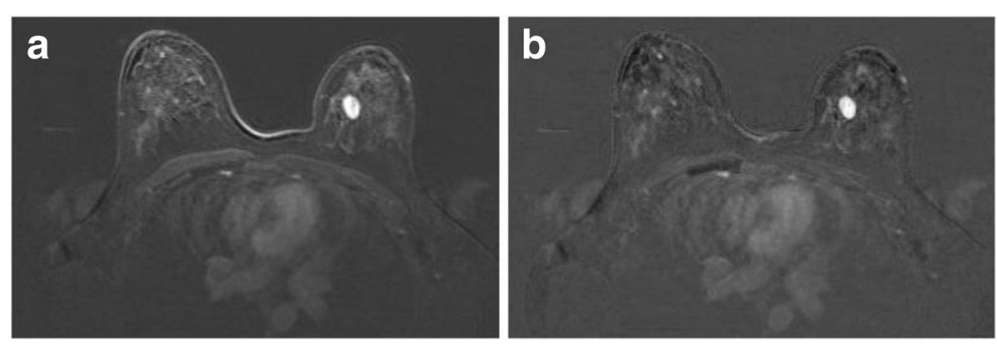

Fig. 2 The influence of motion correction. Subtracted images for a patient with an oval benign lesion in the left breast at the fifth post-contrast time point $\mathbf{a}$ before and $\mathbf{b}$ after applying motion correction step 

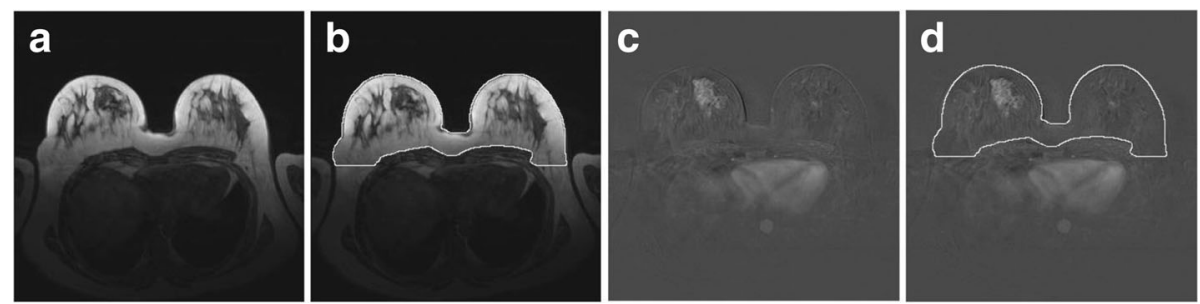

Fig. 3 Demonstration of the breast region segmentation method using sample images. a Nonfat-suppressed image. b Breast segmentation from a. $\mathbf{c}$ The subtracted image at the first post-contrast time point and $\mathbf{d}$ breast segmentation from $\mathbf{c}$

is related to a voxel location. Afterwards, the maximum enhancement ratio is convolved with a Gaussian smoothing filter at 10 exponentially distributed scales between 0 and $10 \mathrm{~mm}$. Because of the various sizes of the breast lesions, different scales are computed and the highest response is chosen for each voxel. Eventually, local maxima of voxel values are found based on a spherical kernel with a radius of $10 \mathrm{~mm}$. The achieved points are potential lesion voxels which are utilized to detect breast lesions. However, the signal intensity of voxels in the blood vessels, noise, skin, and fibroglandular tissues can be similar to those of the lesion voxels. Hence, some of the detected potential lesion voxels do not belong to the lesions. In order to remove these false detections, a combination of FCM clustering and vesselness filter has been used.

FCM clustering technique is utilized to partition the voxels based on the signal intensity variation over time (one pre-contrast and five post-contrast time points) into two categories: lesion and non-lesion. Signal intensity variation over time is one of the tissue characteristics which is widely used for the segmentation and classification of breast lesions [20,21]. Figure 4 presents signal intensity variation over time for some randomly chosen voxels which belong to different lesion and nonlesion tissues. As shown in this figure, the signal intensity variations of voxels in the lesion and non-lesion tissues have different characteristics. Each voxel is represented using signal intensity variation over time as written here,

$$
X=\left\{\mathbf{x}_{i}, i=1,2, \cdots, N \mid \mathbf{x}_{i}=\left(I_{i 0}, I_{i 1}, \cdots, I_{i, T-1}\right)\right\},
$$

where $\mathbf{x}_{i}$ represents the data vector for the $i^{t h}$ voxel, $N$ is the number of voxels, $I_{i t}(t=0,1, \cdots, T-1)$ is the intensity value of the $i^{\text {th }}$ voxel at time point $t$, and $T$ is the number of time points $(T=6)$. FCM clustering process is performed based on minimization of the objective function by iteratively updating the membership functions and the cluster centers. The objective function, cluster centers, and membership functions are defined here [22]:

$$
\begin{aligned}
& F=\sum_{i=1}^{N} \sum_{k=1}^{w} \mu_{k i}^{m}\left\|\mathbf{x}_{\mathbf{i}}-\mathbf{v}_{\mathbf{k}}\right\|^{2} \\
& \mathbf{v}_{\mathbf{k}}=\frac{\sum_{i=1}^{N} \mu_{k i}^{m} \mathbf{x}_{\mathbf{i}}}{\sum_{i=1}^{N} \mu_{k i}^{m}}, k=1,2, \cdots, w, \\
& \mu_{k i}=\frac{1}{\sum_{j=1}^{w}\left\{\frac{\left\|\mathbf{x}_{\mathbf{x}}-v_{k}\right\|}{\left\|\mathbf{x}_{\mathbf{i}}-v_{j}\right\|}\right\}^{\frac{2}{m-1}}}, k=1,2, \cdots ; w ; i=1,2, \cdots ; N .
\end{aligned}
$$

where $m \in[1, \infty)$ and controls the fuzziness of the clustering results, $w$ is the number of clusters, $\mathbf{v}_{\mathbf{k}}$ is the center of the $k^{\text {th }}$ cluster, and $\mu_{k i}$ is the membership values of $i^{\text {th }}$ voxel to $k^{\text {th }}$ cluster which continuously ranges from 0 to 1. Utilizing the class membership values of voxels, two membership matrices are created $\left(\mu_{\text {lesion }}\right.$ and $\left.\mu_{\text {nonlesion }}\right)$. In Fig. 5, the lesion membership matrices are shown for two sample images. Each entry in this matrix represents

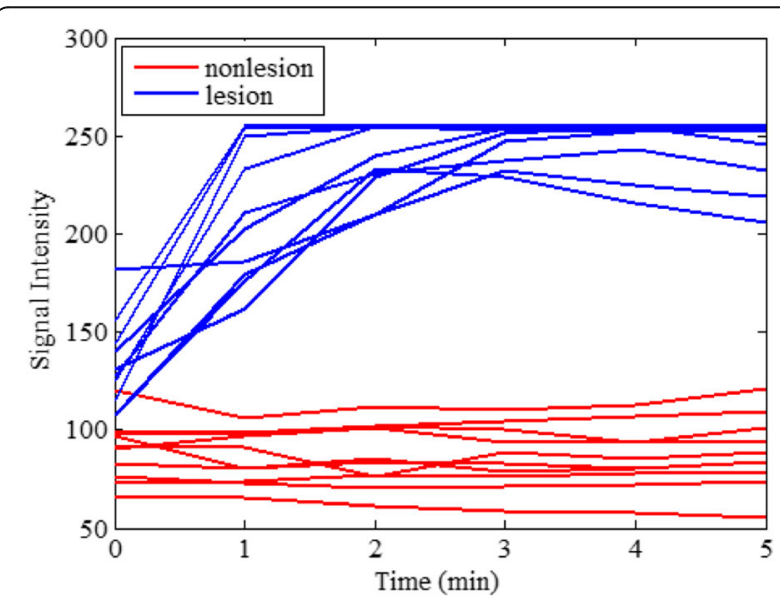

Fig. 4 Comparison of signal intensity variation over time in lesion and non-lesion tissues. Blue and red curves present signal intensity variation over time (one pre-contrast and five post-contrast time points) for twenty voxels which belong to different lesion and non-lesion tissues, respectively 

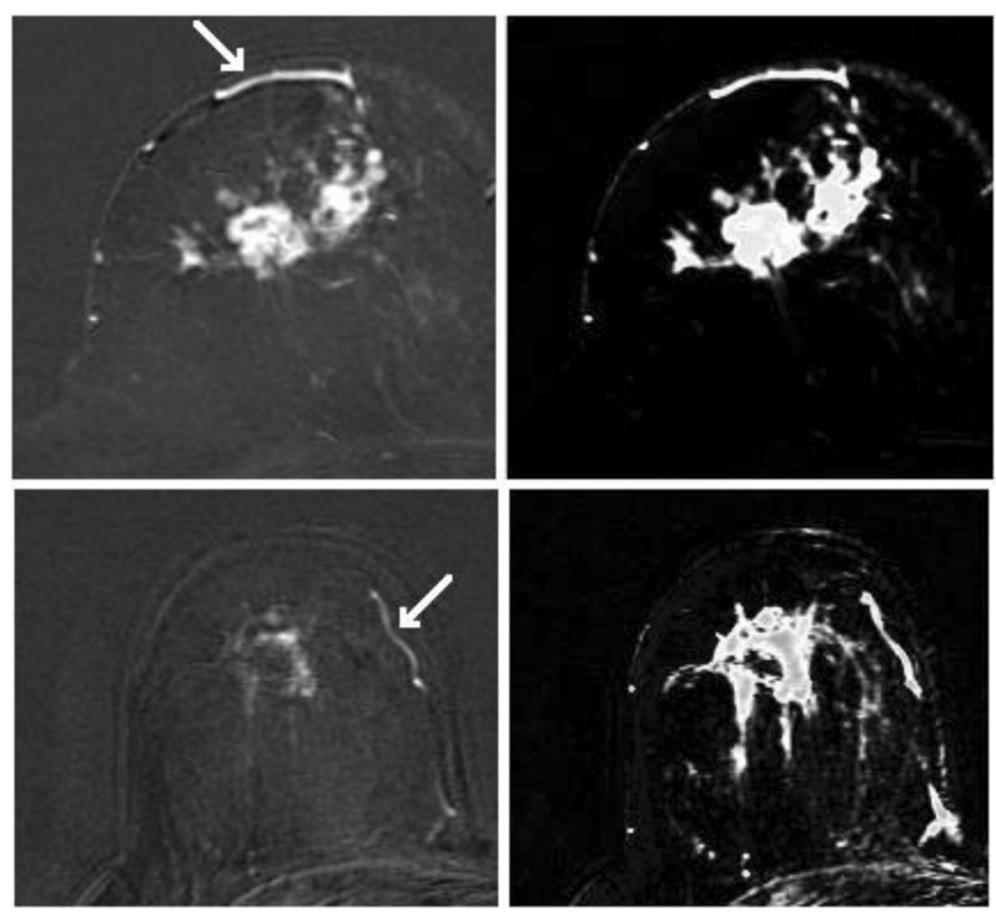

Fig. 5 The clustering results from the FCM method. The first column includes the subtracted image and its threshold at the first post-contrast time point, and the second column provides the lesion membership matrix obtained by FCM clustering. Mammary vessels (arrows) and breast lesions have similar intensity

the degree of similarity between corresponding voxel and lesion tissue. By thresholding the lesion membership matrix, spurious candidate voxels which may belong to noise or normal breast tissues are eliminated. As it can be observed from this figure, the vessels show contrast enhancement similar to the breast lesions. It means that the FCM clustering places the voxels which belong to the mammary vessels and breast lesions in one cluster. Thus, the potential lesion voxels which belong to the vessels should be detected and eliminated to reduce false positive detections.

To identify mammary vessels, we apply Hessian-based filter introduced by Frangi et al. [23] which is one of the most well-known vesselness filters. In the scale space, the second-order derivative of an image $I_{0}(p)=I_{0}(x, y, z)$ is called Hessian matrix and can be obtained by Eq. 6:

$$
H_{\sigma}(p)=\left\{\begin{array}{lll}
\frac{\partial^{2} I_{\sigma}(x, y, z)}{\partial x^{2}} & \frac{\partial^{2} I_{\sigma}(x, y, z)}{\partial x \partial y} & \frac{\partial^{2} I_{\sigma}(x, y, z)}{\partial x \partial z} \\
\frac{\partial^{2} I_{\sigma}(x, y, z)}{\partial y \partial x} & \frac{\partial^{2} I_{\sigma}(x, y, z)}{\partial y^{2}} & \frac{\partial^{2} I_{\sigma}(x, y, z)}{\partial y \partial z} \\
\frac{\partial^{2} I_{\sigma}(x, y, z)}{\partial z \partial x} & \frac{\partial^{2} I_{\sigma}(x, y, z)}{\partial z \partial y} & \frac{\partial^{2} I_{\sigma}(x, y, z)}{\partial z^{2}}
\end{array}\right\}
$$

where $p=(x, y, z)$ is a voxel location and $I_{\sigma}$ is a blurred image at a certain scale defined as:

$$
I_{\sigma}(p)=I_{\sigma}(x, y, z)=I_{0}(x, y, z) \otimes G_{\sigma}(x, y, z)
$$

where $\otimes$ is the convolution operator and $G_{\sigma}(x, y, z)$ is the 3D Gaussian kernel defined as follows:

$$
\begin{aligned}
G_{\sigma}(p) & =G_{\sigma}(x, y, z) \\
& =\frac{1}{\sqrt{2 \pi \sigma^{2}}} \exp -\frac{\left(x^{2}+y^{2}+z^{2}\right)}{2 \sigma^{2}}
\end{aligned}
$$

where $\sigma$ is the standard deviation and has to be set according to the approximate width of the vessels. Eigenvalues of the Hessian matrix present a good geometric interpretation of the image; hence, they are used to detect different structures. In our approach, the eigenvalues of the Hessian matrix are sorted as: $\left|\lambda_{1}\right|<\left|\lambda_{2}\right|<\left|\lambda_{3}\right|$ and according to Eqs. 6-8, they depend on the voxel location and standard deviation. Frangi et al. [23] notify that a voxel belonging to a white vessel on a black background is given by small $\lambda_{1}$ and high negative values of $\lambda_{2}$ and $\lambda_{3}$. The vesselness function is defined here [23] as follows:

$$
v_{o}(p, \sigma)= \begin{cases}0 & \left(1-\exp \left(-\frac{R_{A}^{2}}{2 \alpha^{2}}\right)\right) \exp \left(-\frac{R_{B}^{2}}{2 \beta^{2}}\right) \\ \times\left(1-\exp \left(-\frac{s^{2}}{2 c^{2}}\right)\right) \text { if } \lambda_{2}>0 & \text { or } \lambda_{3}>0 \\ \text { otherwise }\end{cases}
$$



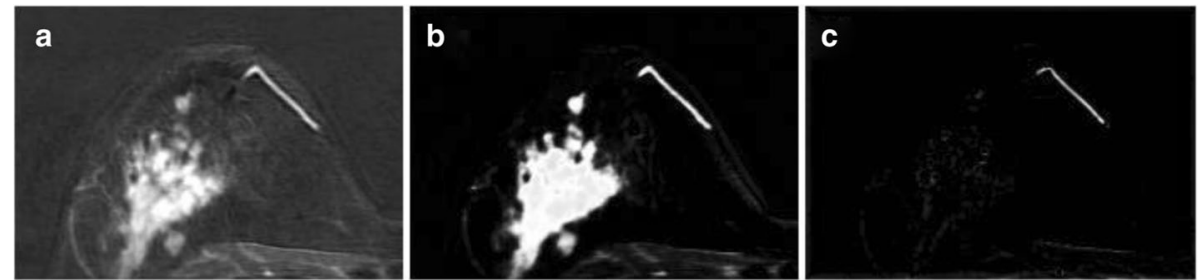

Fig. 6 Result from the vesselness filter. a The subtracted image at the first post-contrast time point. b Lesion membership matrix obtained by FCM clustering. $\mathbf{c}$ Response of the vesselness filter

where $\alpha$ and $\beta$ are fixed to 0.5 and $c$ is the half of the maximum Hessian norm. Moreover, $R_{A}, R_{B}$, and $s$ are given as follows:

$$
\begin{aligned}
& R_{A}=\frac{\left|\lambda_{2}\right|}{\frac{\left|\lambda_{3}\right|}{2}}, R_{B}=\frac{\left|\lambda_{1}\right|}{\sqrt{\left|\lambda_{2} \lambda_{3}\right|}}, s \\
& =\sqrt{\lambda_{1}^{2}+\lambda_{2}^{2}+\lambda_{3}^{2}} \text {. }
\end{aligned}
$$

The term $R_{A}$ distinguishes between plate-like and tubular-like structures, $R_{B}$ describes the deviation from a blob-like structure, and $s$ represents the difference between vessel and background. Due to different diameters of mammary vessels, the vesselness filter is applied at six exponentially distributed scales between the maximum and minimum scales which are $\sigma_{\min }=0.5$ and $\sigma_{\max }=1$ and the highest value is chosen for each voxel.

$$
v_{o}(p)=\max _{\sigma_{\min } \leq \sigma \leq \sigma_{\max }} v_{o}(p, \sigma) .
$$

Vessel detection is performed on the subtracted images at the first post-contrast time point due to the maximum contrast enhancement of vessels in the early frames. The obtained result from the vesselness filter for a sample image is presented in Fig. 6. The value of each entry in the response matrix ranges from 0 to 1 and demonstrates the degree of similarity of each voxel to the vessel.

Lesion membership matrix obtained by the FCM algorithm and the response of the vesselness filter are then thresholded to eliminate the false positive detections in the potential lesion voxel set. The threshold level is chosen equal to 0.5 for both the lesion membership matrix and response of the vesselness filter. Lesion membership matrix is now converted to a binary image which shows normal voxels in black and lesion voxels in white. Also, a binary image is generated for response of the vesselness filter which shows non-vessel voxels in black and mammary vessel voxels in white. Consequently, the voxels with label one from the thresholded lesion membership matrix and label zero from the thresholded response of the vesselness filter are selected as the final potential lesion voxels.

\subsection{Detection of potential lesion regions}

In the previous processing step, the potential lesion voxels are segmented out using a combination of the maximum enhancement ratio, vesselness filter, and FCM clustering. The obtained potential lesion voxels are used as the seed points for the seeded region-growing algorithm to segment the potential lesion regions. The seeded region-growing algorithm is used because it is simple and robust [24]. The seeded region-growing algorithm starts with an initial seed voxel and tries to compare its neighborhood voxels with this seed according to a specific homogeneity criterion and then enlarges the size of the region iteratively. If the neighboring voxel satisfies the homogeneity criterion, it will be joined to the segmented region. Twenty-six neighbors of the new voxel are tested according to the homogeneity criterion, and then, this process will be continued in the same way. The initial seed voxel and homogeneity criterion are usually selected manually [25]. In this study, an automated version of the seeded region-growing algorithm is performed for choosing the parameters. The detected potential lesion voxels are considered as the initial seed voxels, and the attributes which are used to select the potential lesion voxels are considered as the growth criteria of the seeded region-growing algorithm. The neighborhood voxels with label one from the thresholded

\begin{tabular}{|c|c|c|c|c|c|c|c|c|c|c|c|}
\hline Threshold & 0.0 & 0.1 & 0.2 & 0.3 & 0.4 & 0.5 & 0.6 & 0.7 & 0.8 & 0.9 & 1 \\
\hline Detection rate & 0.98 & 0.95 & 0.94 & 0.92 & 0.89 & 0.86 & 0.81 & 0.74 & 0.59 & 0.52 & 0 \\
\hline False positives per case & 13 & 5.91 & 5.29 & 4.41 & 3.53 & 2.80 & 2.20 & 1.40 & 0.72 & 0.37 & 0 \\
\hline Number of undetected lesions & 3 & 8 & 10 & 13 & 18 & 23 & 32 & 44 & 69 & 81 & 170 \\
\hline
\end{tabular}
lesion membership matrix and label zero from the

Table 1 The result of detection rate, false positives, and number of undetected lesions according to the different threshold values 


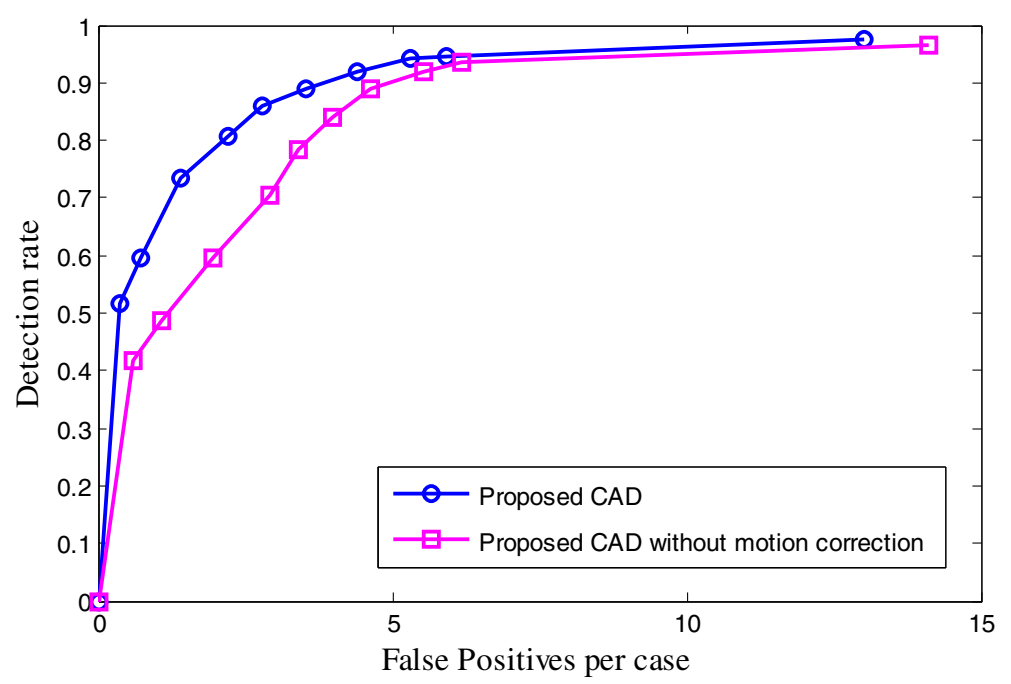

Fig. 7 Comparison of FROC curves for breast lesion detection with and without motion correction

thresholded response of the vesselness filter are considered to be in the potential lesion region.

\subsection{False positive reduction}

Despite the elimination of spurious candidate voxels in Section 3.3, the potential lesion regions are not only breast lesions and there are still some false positive findings. The existence of these false detections can effect on the performance of the CAD system. In order to reduce false positive detections, a discrimination step is used to determine whether a potential lesion region is a true lesion or a false positive detection. This is achieved by classifying the potential lesion regions into two classes, lesion and normal breast tissue, based on the morphological and kinetic features as inputs to the SVM classifier. The main reason for choosing SVM classifier is its high generalization ability, robustness to outliers, and absence of local minima [26].

For classifying the potential lesion regions, morphological and kinetic features are calculated after applying the 3D-connected component algorithm [27, 28] on the potential lesion regions. Morphological features consist of volume, compactness, radius, and spiculation. Lesion volume is utilized to decrease false positive detections, since the majority of the false positive findings have the smaller volumes with respect to the lesions. Compactness describes the correlation between the surface and volume of the segmented regions. Radius and spiculation measure variations of the margins in the segmented regions. More details about the morphological features are

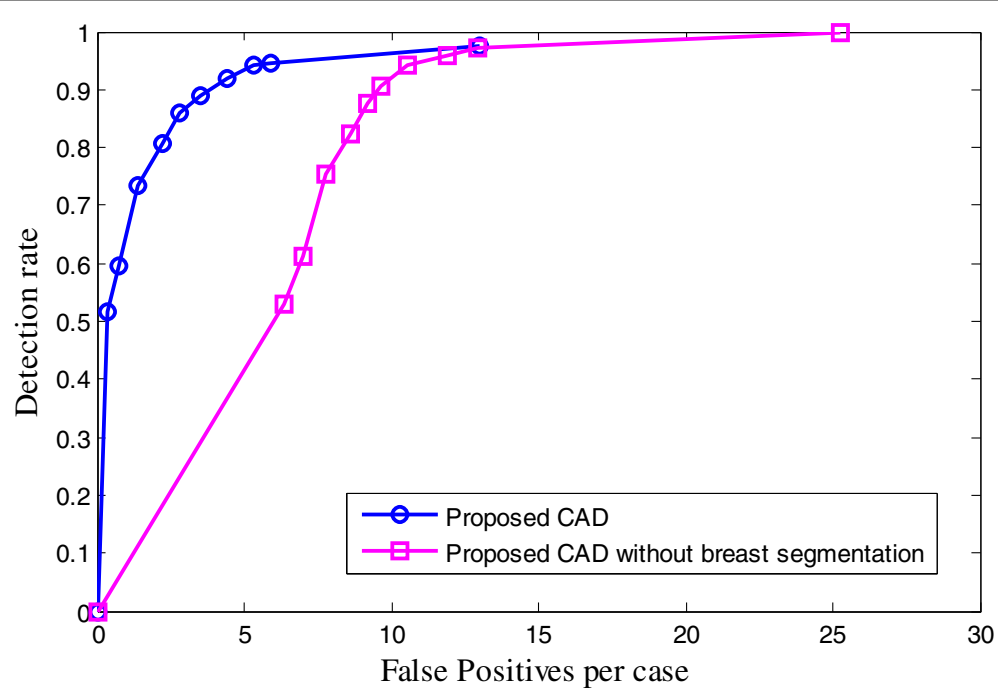

Fig. 8 Comparison of the FROC curves obtained from the proposed CAD with and without breast segmentation process 


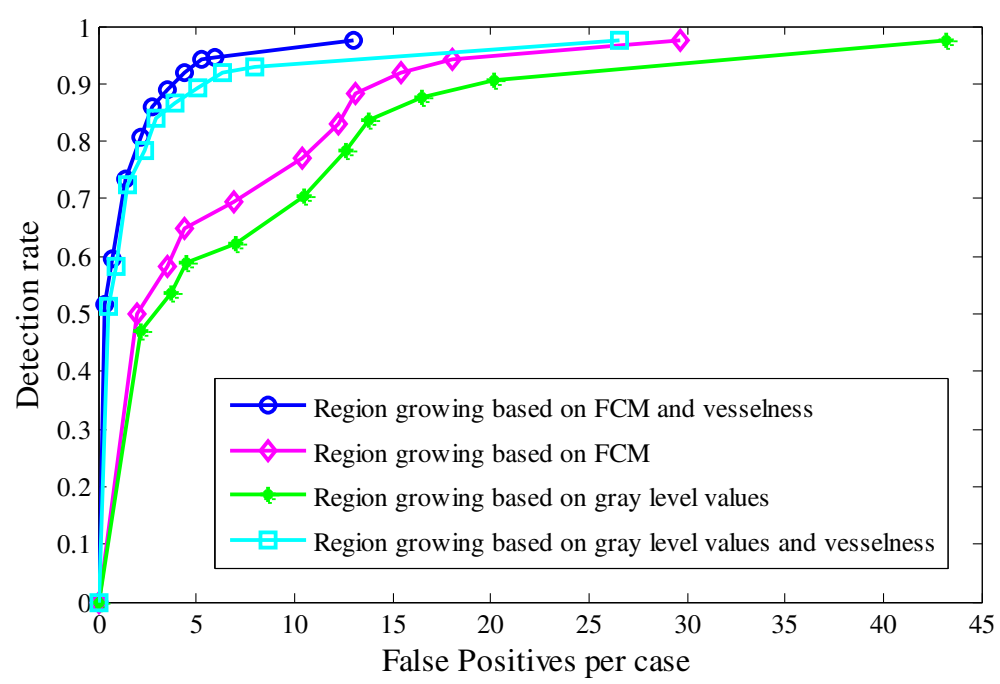

Fig. 9 FROC curves of the proposed CAD system using different region-growing algorithms

described in [21, 29]. To characterize the kinetic features, maximum enhancement (ME), time to peak (TP), uptake rate (UR), washout rate (WR), and area under the curve are extracted from the time-intensity curve of each voxel. These kinetic features are computed according to the relative signal enhancement [14]:

$$
R_{t}=\frac{I_{t}-I_{0}}{I_{0}}
$$

For each voxel in the potential lesion region, ME, TP, UR, and WR are defined here $[14,21]$ as follows:

$$
\begin{aligned}
& \mathrm{ME}=\max _{t=1, \cdots, 5}\left(R_{t}\right), \\
& \mathrm{TP}=\arg \max _{t}\left(R_{t}\right)
\end{aligned}
$$

$$
\begin{aligned}
& \mathrm{UR}=\frac{\mathrm{ME}}{\mathrm{TP}}, \\
& \mathrm{WR}= \begin{cases}\frac{R_{T P}-R_{5}}{5-\mathrm{TP}} & \text { if } \mathrm{TP} \neq 5, \\
0 & \text { if } \mathrm{TP}=5 .\end{cases}
\end{aligned}
$$

For each potential lesion region, 19 features are totally extracted which contain kinetic parameters for seed point of the segmented region, the average and standard deviation of the kinetic parameters for the entire voxels in the segmented region, and four morphological features. Each potential lesion region is classified by feeding its feature vector as the input to the SVM classifier. More details about the SVM is available in [26].

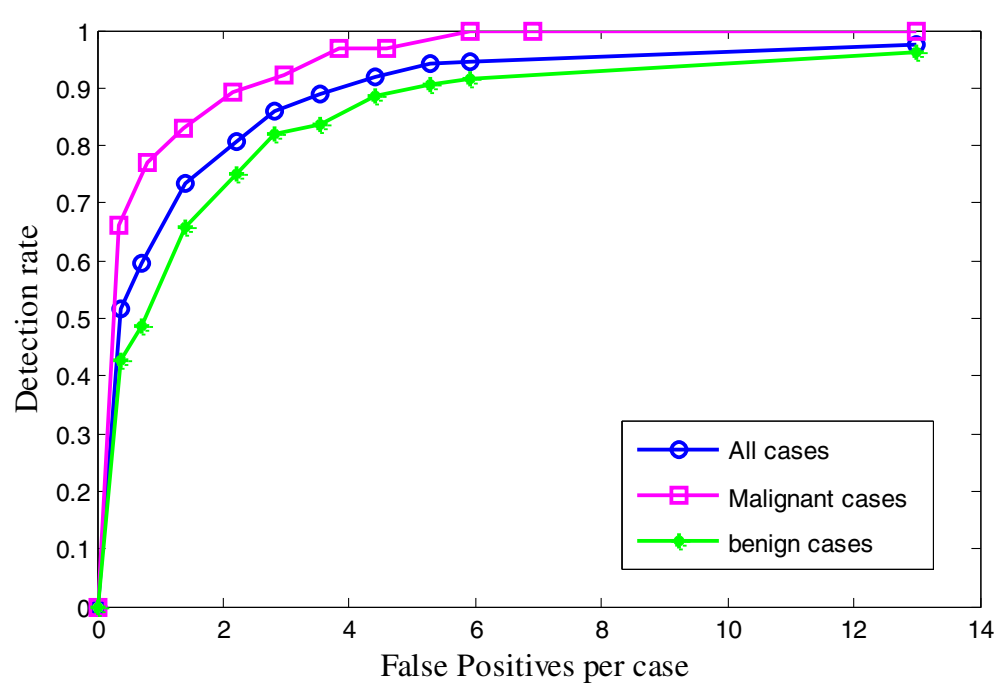

Fig. 10 The performance of the proposed CAD system for benign and malignant lesions 

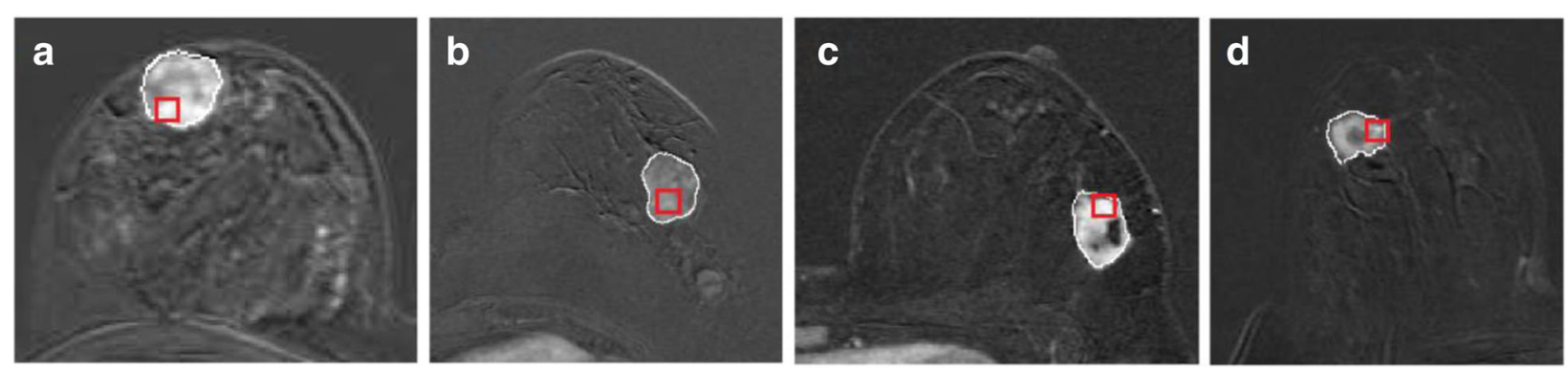

Fig. 11 Detected benign and malignant lesions by the proposed CAD system. a, b Benign lesions. c-d Malignant lesions on the subtracted images at the first post-contrast time point. The squared markers refer to the potential lesion voxels obtained by the presented method. These potential lesion voxels are the seed points for the region growing algorithm. The connected component boundaries of the legions are properly delineated

\section{Results and discussion}

In this study, 76 cases are utilized to evaluate the performance of the proposed CAD system. A fivefold crossvalidation is performed to achieve unbiased detection results. The number of lesions is approximately considered similar for all folds. The SVM classifier distinguishes true lesion region from the false one in the false positive reduction stage. In order to find an optimal configuration for the classifier, we investigate linear SVM and nonlinear SVM with polynomial and RBF kernels with different parameters [26]. The parameters that should be set for the classifier are cost $C$, polynomial degree $d$, and kernel scale $\gamma$. Cost $C$ ranges between $2^{-i}$ and $2^{i}, i$ $=[0,5,10]$. Kernel scale $\gamma$ ranges between $2^{-i}$ and $2^{i}, i$ $=[0,5,10]$. In addition, polynomial degree $d$ varies between 1 and 5 . We have experimentally found that the highest averaged accuracy can be achieved using the RBF kernel with $c=2^{10}$ and $\gamma=2^{-5}$.

Two quality metrics, the detection rate and false positive per case, have been used to evaluate the results of the lesion detection process. Detection rate is the number of true positive detections divided by the total number of lesions, and false positive rate per case is the number of false positive detections divided by the total number of cases. A plot of detection rate vs. false positive rate per case, by changing the decision threshold, provides the FROC curve [30]. In the false positive reduction step, SVM assigns a probability value to each potential lesion region. If the assigned probability value is larger than or equal to the threshold value, the potential lesion region is classified as a true lesion region. By changing the threshold level between 0 and 1 with a step of 0.1 , FROC curves can be generated. Table 1 shows the obtained values of detection rate, false positives per case, and number of undetected lesions according to the different threshold values.

The influence of the motion correction process on the performance of the proposed CAD system is investigated primarily. Figure 7 shows the FROC curves obtained for breast lesion detection with and without applying motion correction. As it can be observed from Fig. 7, the motion correction process offers a better performance for detecting breast lesions.

In the proposed CAD system, the breast region is automatically segmented to avoid false detections caused by enhancing tissues of the heart and vessels outside of

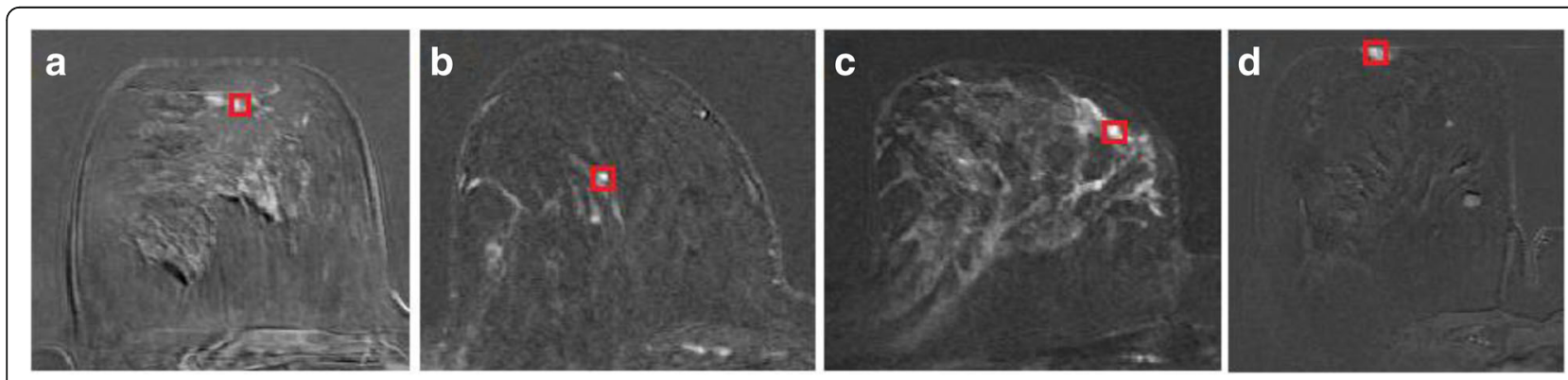

Fig. 12 False positive detections on the subtracted images for four different normal cases. a-c Three normal cases without breast lesions, there are contrast enhancement in fibroglandular tissues because of injection of contrast agent, and $\mathbf{d}$ a normal case without breast lesion, there is contrast enhancement in the nipple because of injection of contrast agent. The squared markers refer to the potential lesion voxels obtained by the presented method. Due to small diameter of false positive detections, the connected component boundaries are shown very unnoticeable. For such cases, only the region that the potential legion voxels are located, are demonstratd on the figures 
Table 2 Performance comparison between the proposed CAD system and available methods

\begin{tabular}{llll}
\hline Methods & Detection rate & False positive detections & Database \\
\hline Ertas et al. [8] & 1 & 0.64/case & 19 case (19 benign and 20 malignant lesions) \\
Vignati et al. [10] & 0.89 & 4/breast & 48 cases (12 benign and 53 malignant lesions) \\
Renz et al. [9] & 0.96 & $0.16 /$ case & 108 cases (53 benign and 88 malignant lesions) \\
Chang et al. [11] & 0.93 & 6.15/case & 54 cases (28 benign and 67 malignant lesions) \\
Gubern-Mérida et al. [14] & 0.89 & 4/normal case & 95 cases (105 malignant lesions and 114 normal cases) \\
The Proposed method & 0.94 & 5.29/case & 76 cases (107 benign and 63 malignant lesions) \\
\hline
\end{tabular}

the tested breast. Figure 8 shows FROC curves obtained from the proposed CAD with and without the breast segmentation step. The breast segmentation process improves the performance of the CAD system by reducing false positive detections.

An accurate segmentation of the potential lesion regions plays an important role in a CAD system because it influences the values of the extracted features and accuracy of the classifier to reduce false positive findings. In this work, we introduce the seeded region-growing algorithm where the initial seed point and homogeneity criterion are selected based on the FCM and vesselness filter. The performance of the proposed CAD system is evaluated using different criteria for the seeded regiongrowing algorithm. The proposed seeded region-growing algorithm is compared with different region-growing algorithms with and without vesselness filter and applying only FCM. FROC curves obtained from different regiongrowing algorithms are shown in Fig. 9. It is clear that the proposed region-growing algorithm based on vesselness filter and FCM provides higher detection rates at the lower false positives per case in comparison with other region-growing algorithms.

Figure 10 illustrates FROC curves obtained for malignant, benign, and all lesions. The presented CAD system has better performance for detecting malignant lesions. Due to similar features of benign lesions and some normal tissues such as vessels, fibroglandular, and skin, distinguishing benign lesions is a challenging process. Some examples of benign and malignant lesions detected by the proposed CAD system are shown in Fig. 11. Also, some selected samples of the false positive detections in normal cases are shown in Fig. 12.

To date, only a few studies have investigated automatic detection of breast lesions in DCE-MRI. Table 2 summarizes the accuracy for the proposed CAD system in comparison with other related works. It is worth mentioning that their own datasets have been employed for the evaluation procedures. Ertas et al. [8] obtain higher performance metric values than others reported in the literature. However, their approach is validated on a smaller number of cases compared to other studies. Gubern-Mérida et al. [14] validate their method on 209 MRI cases, which is larger than the other datasets. However, the mentioned dataset does not include benign lesions while the discrimination of benign lesions from the normal breast tissues is one of the challenging issues in a CAD system. Among the mentioned studies, only GubernMérida et al. [14] and Chang et al. [11] present their results based on FROC analysis. Vignati et al. [10] and Renz et al. [9] do not evaluate the performance of their methods on non-mass-like enhancement lesions. Chang et al. [11] and Ertas et al. [8] do not clearly express whether their evaluations are performed on non-mass-like lesions.

\section{Conclusions}

In this paper, a CAD system is proposed for detecting breast lesions in DCE-MRI. The contribution of the CAD system is to detect potential lesion regions using a region-growing algorithm with new criteria based on FCM clustering and vesselness filter. Moreover, 3D morphological characteristics and kinetic features are utilized to eliminate the spurious candidate regions. The proposed algorithm is applied to the prepared dataset (IUST-BREASTMRI-1) with and without motion correction. Additionally, the effect of using the breast segmentation algorithm is investigated. The qualitative and quantitative results indicate that the performance of our CAD system has been improved significantly by applying the motion correction and breast segmentation steps. Future research will aim at improving the performance of the proposed CAD system by reducing false positives findings and increasing the ability of approach to separate benign lesions and normal breast tissues.

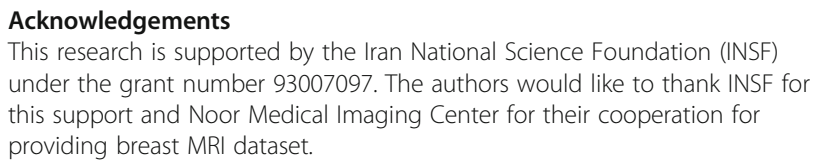

Competing interests

The authors declare that they have no competing interests.

\section{Publisher's Note}

Springer Nature remains neutral with regard to jurisdictional claims in published maps and institutional affiliations. 


\section{Author details}

'School of Electrical Engineering, Iran University of Science and Technology (IUST), Tehran, Iran. ${ }^{2}$ Advanced Diagnostic \& Interventional Radiology Research Center (ADIR), Tehran University of Medical Sciences, Tehran, Iran.

Received: 24 October 2016 Accepted: 12 May 2017

Published online: 25 May 2017

\section{References}

1. American Cancer Society, Breast Cancer Facts \& Figures 2013-2014. (Atlanta, 2013)

2. D Saslow, C Boetes, W Burke, S Harms, MO Leach, CD Lehman, EA Morris, E Pisano, M Schnall, S Sener, RA Smith, E Warner, M Yaffe, KS Andrews, CA Russell, American Cancer Society Guidelines for breast screening with MRI as an adjunct to mammography. CA-Cancer J. Clin. 57, 75-89 (2007)

3. EA Morris, L Liberman, Breast MRI: Diagnosis and Intervention (SpringerVerlag, New York, 2005)

4. A Fausto, A Magaldi, B Babaei Paskeh, L Menicagli, EN Lupo, F Sardanelli, MR imaging and proton spectroscopy of the breast: how to select the images useful to convey the diagnostic message. Radiol Med 112, 1060-1068 (2007)

5. S Behrens, H Laue, M Althaus, T Bohler, B Kuemmerlen, HK Hahn, HO Peitgen, Computer assistance for MR based diagnosis of breast cancer: present and future challenges. Comput. Med. Imag. Graph. 31, 236-247 (2007)

6. S Kligerman, L Cai, CS White, The effect of computer-aided detection on radiologist performance in the detection of lung cancers previously missed on a chest radiograph. J. Thorac. Imag. 28, 244-252 (2013)

7. LWW Burhenne, SA Wood, CJ D'Orsi, SA Feig, DB Kopans, KF O'Shaughnessy, EA Sickles, L Tabar, CJ Vyborny, RA Castellino, Potential contribution of computer-aided detection to the sensitivity of screening mammography. Radiology 215, 554-562 (2000)

8. G Ertas, HO Gulcur, O Osman, ON Ucan, M Tunaci, M Dursun, Breast MR segmentation and lesion detection with cellular neural networks and 3D template matching. Comput. Biol. Med. 38, 116-126 (2008)

9. DM Renz, J Böttcher, F Diekmann, A Poellinger, MH Maurer, A Pfeil, F Streitparth, F Collettini, U Bick, B Hamm, EM Fallenberg, Detection and classification of contrast-enhancing masses by a fully automatic computerassisted diagnosis system for breast MRI. J. Magn. Reson. Imaging 35, 10771088 (2012)

10. A Vignati, V Giannini, M De Luca, L Morra, D Persano, LA Carbonaro, I Bertotto, L Martincich, D Regge, A Bert, F Sardanelli, Performance of a fully automatic lesion detection system for breast DCE-MRI. J. Magn. Reson. Imaging. 34, 1341-1351 (2011)

11. YC Chang, YH Huang, CS Huang, JH Chen, RF Chang, Computerized breast lesions detection using kinetic and morphologic analysis for dynamic contrast-enhanced MRI. Magn. Reson. Imaging. 32, 514-522 (2014)

12. EA Morris, CE Comstock, CH Lee, ACR BI-RADS magnetic resonance imaging, in ACR BI-RADS Atlas: Breast Imaging Reporting and Data System (American College of Radiology, Reston, 2013)

13. RM Mann, J Veltman, H Huisman, C Boetes, Comparison of enhancement characteristics between invasive lobular carcinoma and invasive ductal carcinoma. J. Magn. Reson. Imaging 34, 293-300 (2011)

14. A Gubern-Mérida, R Martí, J Melendez, JL Hauth, RM Mann, N Karssemeijer B Platel, Automated localization of breast cancer in DCE-MRI. Med. Image Anal. 20, 265-274 (2015)

15. PA Yushkevich, J Piven, HC Hazlett, RG Smith, S Ho, JC Gee, G Gerig, Userguided 3D active contour segmentation of anatomical structures: significantly improved efficiency and reliability. Neuroimage. 31, 1116-1128 (2006)

16. D Rueckert, LI Sonoda, C Hayes, DLG Hill, MO Leach, DJ Hawkes, Nonrigid registration using free-form deformations: application to breast MR images. IEEE Trans. Image Process. 18, 712-721 (1999)

17. P Viola, WM Wells, Alignment by maximization of mutual information. Int. J. Comput. Vision. 24, 137-154 (1997)

18. A Myronenko, X Song, Intensity-based image registration by minimizing residual complexity. IEEE Trans. Med. Imaging. 29, 1882-1891 (2010)

19. A Fooladivanda, SB Shokouhi, N Ahmadinejad, MR Mosavi, Automatic Segmentation of Breast and Fibroglandular Tissue in Breast MRI using Local Adaptive Thresholding. Paper presented at the 21th Iranian Conference on Biomedical Engineering (ICBME 2014), Amirkabir University of Technology (Tehran Polytechnic), Tehran, Iran, 26-28 Nov 2014.
20. W Chen, ML Giger, U Bick, A fuzzy C-means (FCM) based approach for computerized segmentation of breast lesions in dynamic contrast-enhanced MR images. Acad. Radiol. 13, 63-72 (2006)

21. WJ Chen, ML Giger, U Bick, GM Newstead, Automatic identification and classification of characteristic kinetic curves of breast lesions on DCE-MRI. Med. Phys 33, 2878-2887 (2006)

22. JC Bezdek, Pattern Recognition with Fuzzy Objective Function Algorithm (Plenum Press, New York, 1981)

23. AF Frangi, WJ Niessen, KL Vincken, MA Viergever, Multiscale vessel enhancement filtering, in Medical Image Computing and ComputerAssisted Intervention (MICCAI'98), ed. by WM Wells, A Colchester, SL Delp. Lecture Notes in Computer Science, vol. 1496 (Springer Verlag, Berlin, 1998), pp. 130-137.

24. R Adams, L Bischof, Seeded region growing. IEEE Trans. Pattern Anal. Machine Intell. 16, 641-647 (1994)

25. LA Meinel, AH Stolpen, KS Berbaum, LL Fajardo, JM Reinhardt, Breast MRI lesion classification: improved performance of human readers with a backpropagation neural network computer-aided diagnosis (CAD) system. J. Magn. Reson. Imaging. 25, 89-95 (2007)

26. S Abe, Support Vector Machines for Pattern Classification (Springer Verlag, London, 2005)

27. A Rosenfel, Connectivity in digital pictures. J. ACM. 17, 146-147 (1970)

28. A Rosenfel, JL Pfaltz, Sequential operations in digital picture processing. J. ACM. 13, 471-477 (1966)

29. YH Huang, YC Chang, CS Huang, TJ Wu, JH Chen, RF Chang, Computeraided diagnosis of mass-like lesion in breast MRI: differential analysis of the 3-D morphology between benign and malignant tumors. Comput. Meth. Prog. Bio. 112, 508-517 (2013)

30. SY Yu, L Guan, A CAD system for the automatic detection of clustered microcalcifications in digitized mammogram films. IEEE Trans. Med. Imaging. 19, 115-126 (2000)

\section{Submit your manuscript to a SpringerOpen ${ }^{\circ}$ journal and benefit from:}

- Convenient online submission

- Rigorous peer review

- Open access: articles freely available online

- High visibility within the field

- Retaining the copyright to your article

Submit your next manuscript at springeropen.com 